\title{
Non-Coherent Amplify-and-Forward Generalized Likelihood Ratio Test Receiver
}

\author{
Michael R. Souryal \\ National Institute of Standards and Technology \\ Advanced Network Technologies Division \\ Gaithersburg, Maryland, USA
}

\begin{abstract}
This paper proposes a simple non-coherent amplifyand-forward receiver for the relay channel and evaluates its diversity performance for Rayleigh fading channels. We use the generalized likelihood ratio test to obtain the decision rule in closed form, independent of the fading distribution. The receiver is developed for $M$-ary orthogonal signals and multiple relays. The only side information required is the average noise energy at the receiver; no statistical knowledge of the channel gains is needed. We develop closed-form upper and lower bounds on the probability of error of this receiver for the case of binary signaling with a single relay and show that this receiver achieves near-full diversity, with the probability of error decreasing with increasing signal-to-noise ratio (SNR) as $\log ^{2}(\mathrm{SNR}) / \mathrm{SNR}^{2}$ for large SNR. Additional results obtained by simulation demonstrate increasing diversity gain with additional relays.
\end{abstract}

\section{INTRODUCTION}

Various relaying protocols have been proposed as a means for obtaining spatial diversity in wireless fading channels through sharing the antennas of multiple terminals, also known as cooperative diversity. Among the protocols that have been shown to achieve diversity, amplify-and-forward-whereby a relay simply amplifies its received signal from the source-is attractive in that it requires no channel decoding at the relay and no adaptation to the channel to decide whether or not to relay.

Much of the previous work on amplify-and-forward cooperation has been done for coherent receivers (e.g., [1]-[3]), which not only require tracking of the signal phase but also channel state information at the destination of the source-relaydestination concatenated channel. Relatively little attention has been given to non-coherent amplify-and-forward reception, likely due to the absence of a closed-form expression for the maximum-likelihood receiver [4], [5].In [4], a suboptimal receiver inspired by maximal ratio combining was observed to perform worse than non-cooperative transmission. The suboptimum receiver proposed in [5] for binary signals performs comparably to the optimum receiver but requires knowledge at the destination of the average channel gains of all links in the system, which may not be readily available in practice. Likewise, the differential modulation receiver of [6] requires the average gains of the source-relay and relay-destination links.

In this paper, we develop a non-coherent amplify-andforward receiver using the generalized likelihood ratio (GLR) test procedure [7] to obtain the decision rule in closed form,

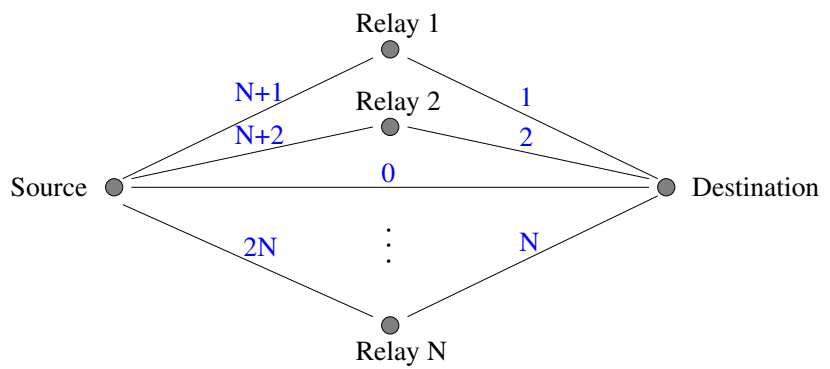

Fig. 1. Sample topology of source, destination and $N$ relays

independent of the fading distribution. Different from previous work, the proposed receiver does not require any statistical knowledge of the channel gains in the system and only requires the local average noise energy. The general receiver applies to $M$-ary orthogonal signal sets used with one or more noninterfering, half-duplex relays. We develop upper and lower bounds on the probability of error in closed form for the case of a single relay, binary signaling, and Rayleigh fading channels, and we show that this receiver achieves nearly full, second-order diversity. Specifically, the error probability of this receiver decreases with increasing signal-to-noise ratio (SNR) as $\log ^{2}$ (SNR) $/ \mathrm{SNR}^{2}$ for large SNR. Additional results obtained by simulation demonstrate higher-order diversity with increasing numbers of relays.

After describing the system model in Section II, we develop the GLR test receiver in Section III. Error probability bounds are derived in Section IV, and numerical results are presented in Section V.

\section{SySTEM MODEL}

The source broadcasts a message to $N$ relays and a destination (see Fig. 1) in the first of $N+1$ orthogonal subchannels. Each relay amplifies and forwards its noisy received signal to the destination in its own subchannel. While this relaying scheme incurs a factor $N+1$ bandwidth expansion or rate reduction, our focus is on the potential diversity gain of this simple approach. The source uses an $M$-ary orthogonal signal set such as $M$-ary frequency shift keying (FSK). The destination employs non-coherent reception (a bank of $M$ noncoherent correlators) and combines the received signals from the source and relays to make a decision as to which of the $M$ symbols was transmitted. 
The channel attenuates each transmission with a random gain and phase rotation and adds noise. Using a discrete-time model representing the sampled outputs of the correlators, the received signals at the relays and destination are

$$
\begin{aligned}
& \mathbf{y}_{0}=\mathbf{A}_{0} \mathbf{x}_{m}+\mathbf{z}_{0} \quad \text { (src to dest) } \\
& \mathbf{y}_{r+N}=\mathbf{A}_{r+N} \mathbf{x}_{m}+\mathbf{z}_{r+N} \quad(\text { src to relay } r) \\
& \mathbf{y}_{r}=\mathbf{A}_{r} \beta_{r} \mathbf{y}_{r+N}+\mathbf{z}_{r} \quad \text { (relay } r \text { to dest) } \\
& =\beta_{r} \mathbf{A}_{r} \mathbf{A}_{r+N} \mathbf{x}_{m}+\beta_{r} \mathbf{A}_{r} \mathbf{z}_{r+N}+\mathbf{z}_{r}
\end{aligned}
$$

where $r=1, \ldots, N$, and the subscripts correspond to the link numbering shown in Fig. 1. The $m$ th element of the $M \times 1$ vector $\mathbf{y}_{l}$ is a complex number whose real and imaginary parts represent the phase quadrature outputs of the $m$ th correlator. The transmitted symbol with phase offset $\phi$ is represented by the $M \times 1$ vector $\mathbf{x}_{m}, m=1, \ldots, M$, having $\sqrt{E_{s}} e^{j \phi}$ as its $m$ th element and 0 as its other elements.

The channel gain of link $l, \mathbf{A}_{l}$, is an $M \times M$ matrix containing random complex channel gains on its main diagonal and 0 at its other elements. ${ }^{1}$ Modeling additive white Gaussian noise at the output of each correlator, the elements of $\mathbf{z}_{l}$ are independent zero-mean, circularly symmetric complex Gaussian random variables with variance $\sigma^{2}$. Furthermore, the channel gains $\mathbf{A}_{l}$ and noise vectors $\mathbf{z}_{l}$ are assumed mutually independent over all $l=0,1, \ldots, 2 N$.

The amplification gain used by relay $r, \beta_{r}$, is typically chosen such that the energy transmitted by the relay satisfies some constraint. For example, to ensure the relay transmits with fixed energy equal to that of the source during each symbol period (i.e., an instantaneous power constraint), then the relay gain would be set as

$$
\beta_{r}=\sqrt{\frac{E_{s}}{\mathbf{y}_{r+N}^{\mathrm{H}} \mathbf{y}_{r+N}}} .
$$

Relay gain (2) varies with the source-relay channel gain and noise realization. An alternative fixed relay gain which satisfies a long-term power constraint for ergodic channels, but which requires the relay to estimate its average received energy, is given by

$$
\beta_{r}=\sqrt{\frac{E_{s}}{\mathrm{E}\left[\mathbf{y}_{r+N}^{\mathrm{H}} \mathbf{y}_{r+N}\right]}}
$$

where $\mathrm{E}[\cdot]$ is the statistical expectation operator. While the proposed receiver is developed below assuming the fixed relay gain (3), the impact of using variable relay gain (2) is discussed in Section V.

\section{GLR TEST RECEIVER}

In general, the destination's maximum-likelihood decision rule as a function of its $N+1$ channel outputs is the following:

$$
\hat{m}=\arg \max _{m=1, \ldots, M} f\left(\mathbf{y}_{0}, \mathbf{y}_{1}, \ldots, \mathbf{y}_{N} \mid \mathbf{x}_{m}\right)
$$

\footnotetext{
${ }^{1}$ Such a model permits, for example, frequency-selective FSK channels.
}

where $f\left(\cdot \mid \mathbf{x}_{m}\right)$ is the conditional density of the channel outputs given that the source transmitted $\mathbf{x}_{m}$. Since, conditioned on $\mathbf{x}_{m}$, the channel outputs are independent, (4) can be expressed as

$$
\begin{aligned}
\hat{m} & =\arg \max _{m=1, \ldots, M} \prod_{l=0}^{N} f\left(\mathbf{y}_{l} \mid \mathbf{x}_{m}\right) \\
& =\arg \max _{m=1, \ldots, M} \sum_{l=0}^{N} \log f\left(\mathbf{y}_{l} \mid \mathbf{x}_{m}\right) .
\end{aligned}
$$

The conventional approach to obtaining these conditional densities requires knowledge of the distribution of the channel gain. Furthermore, a closed-form expression for the conditional density of the relay-destination channel outputs is not available, even for the case of Rayleigh fading [4], [5]. To circumvent these difficulties and to generalize the detector beyond a given fading distribution, we consider the use of the generalized likelihood ratio test to derive the decision rule. In a GLR test, the likelihood function for each hypothesis is evaluated using the maximum-likelihood estimate of the unknown parameter [7, p. 92] (the channel gain, in this case). In other words, the likelihood function is maximized over the unknown parameter.

To apply the GLR test to our problem, we begin with the density of the channel outputs conditioned on both the transmitted signal and the channel gains. Since the channels are independent, we may apply the GLR test to each channel likelihood separately. From (1) we observe that, conditioned on $\mathbf{A}_{0}$ and $\mathbf{x}_{m}, \mathbf{y}_{0}$ is $M$-dimensional Gaussian with mean vector $\mathbf{A}_{0} \mathbf{x}_{m}$ and covariance matrix $\sigma^{2} \mathbf{I}_{M}$, where $\mathbf{I}_{M}$ is the $M \times M$ identity matrix. If we denote the $k$ th element of the diagonal of $\mathbf{A}_{l}$ as $\alpha_{l, k}=a_{l, k} e^{j \theta_{l, k}}$ and the $k$ th element of $\mathbf{y}_{l}$ as $y_{l, k}=w_{l, k} e^{j \psi_{l, k}}$, then the conditional density of $\mathbf{y}_{0}$ is

$$
\begin{aligned}
f\left(\mathbf{y}_{0} \mid \mathbf{x}_{m}, \mathbf{A}_{0}\right) & \\
= & \frac{1}{\left(\pi \sigma^{2}\right)^{M}} \\
& \times \exp \left(-\frac{\left|y_{0, m}-\alpha_{0, m} \sqrt{E_{s}} e^{j \phi}\right|^{2}+\sum_{k \neq m}\left|y_{0, k}\right|^{2}}{\sigma^{2}}\right) .
\end{aligned}
$$

Then, maximizing the source-destination likelihood (6) over $\alpha_{0, m}$ gives

$$
\begin{aligned}
\hat{f}\left(\mathbf{y}_{0} \mid \mathbf{x}_{m}\right) & =\max _{\alpha_{0, m}} f\left(\mathbf{y}_{0} \mid \mathbf{x}_{m}, \alpha_{0, m}\right) \\
& =\frac{1}{\left(\pi \sigma^{2}\right)^{M}} \exp \left(-\frac{\sum_{k \neq m} w_{0, k}^{2}}{\sigma^{2}}\right) \\
& =\frac{1}{\left(\pi \sigma^{2}\right)^{M}} \exp \left(-\frac{\left\|\mathbf{y}_{0}\right\|^{2}-w_{0, m}^{2}}{\sigma^{2}}\right)
\end{aligned}
$$

where $\hat{f}(\cdot)$ is used to refer to the maximized likelihood and is sometimes referred to as the profile or concentrated likelihood.

Similarly, each relay-destination channel output, $\mathbf{y}_{r}, r=$ $1, \ldots, N$, conditioned on $\mathbf{A}_{r}, \mathbf{A}_{r+N}$, and $\mathbf{x}_{m}$, is $M-$ dimensional Gaussian with mean vector $\beta_{r} \mathbf{A}_{r} \mathbf{A}_{r+N} \mathbf{x}_{m}$ and 
covariance matrix having the $k$ th element on its main diagonal as $\tilde{\sigma}_{r, k}^{2}=\sigma^{2}\left(\beta_{r}^{2} a_{r, k}^{2}+1\right)$ and 0 at its other elements. The conditional density of $\mathbf{y}_{r}$, then, is

$$
\begin{aligned}
& f\left(\mathbf{y}_{r} \mid \mathbf{x}_{m}, \mathbf{A}_{r}, \mathbf{A}_{r+N}\right) \\
& =\frac{1}{\pi \tilde{\sigma}_{r, m}^{2}} \exp \left(-\frac{\left|y_{r, m}-\beta_{r} \alpha_{r, m} \alpha_{r+N, m} \sqrt{E_{s}} e^{j \phi}\right|^{2}}{\tilde{\sigma}_{r, m}^{2}}\right) \\
& \quad \times \prod_{k \neq m} \frac{1}{\pi \tilde{\sigma}_{r, k}^{2}} \exp \left(-\frac{w_{r, k}^{2}}{\tilde{\sigma}_{r, k}^{2}}\right) .
\end{aligned}
$$

The relay-destination conditional likelihood (8) depends on both $\alpha_{r, m}$ and $\alpha_{r+N, m}$. Maximizing it with respect to $\alpha_{r+N, m}$, first, results in

$$
\hat{f}\left(\mathbf{y}_{r} \mid \mathbf{x}_{m}, \mathbf{A}_{r}\right)=\frac{1}{\pi \tilde{\sigma}_{r, m}^{2}} \prod_{k \neq m} \frac{1}{\pi \tilde{\sigma}_{r, k}^{2}} \exp \left(-\frac{w_{r, k}^{2}}{\tilde{\sigma}_{r, k}^{2}}\right) .
$$

Recalling that $\tilde{\sigma}_{r, k}^{2}=\sigma^{2}\left(\beta_{r}^{2} a_{r, k}^{2}+1\right)$, we can maximize each factor of the above with respect to $a_{r, k}$ separately. The ML estimate of $a_{r, m}$ is $\hat{a}_{r, m}=0$. The ML estimates of $a_{r, k}, k \neq$ $m$, can be shown to satisfy $\beta_{r}^{2} \hat{a}_{r, k}^{2}+1=w_{r, k}^{2} / \sigma^{2}$. Substituting these estimates into (9) gives

$$
\begin{aligned}
\hat{f}\left(\mathbf{y}_{r} \mid \mathbf{x}_{m}\right) & =\frac{e}{(\pi e)^{M} \sigma^{2}} \prod_{k \neq m} \frac{1}{w_{r, k}^{2}} \\
& =\frac{e}{(\pi e)^{M} \sigma^{2}}\left(\prod_{k=1}^{M} \frac{1}{w_{r, k}^{2}}\right) w_{r, m}^{2} .
\end{aligned}
$$

Finally, using (7) and (10) in place of the likelihood functions in (5) and simplifying gives the following GLR test receiver:

$$
\hat{m}=\arg \max _{m=1, \ldots, M} \frac{w_{0, m}^{2}}{\sigma^{2}}+\sum_{r=1}^{N} \log w_{r, m}^{2} .
$$

We note that since the overall noise variance of the relaydestination channel output depends on the relay-destination channel amplitude (due to noise amplification), elimination of the amplitude parameters also eliminates, as a by-product, the dependence on the amplified noise at the relay. Thus, the only side information required is the variance of the noise local to the destination. We also observe that the sourcedestination contribution to the overall decision metric is linear in the magnitude-squared correlator outputs while the relaydestination contribution is logarithmic, suggesting the weaker reliability of the noise-amplified relayed signals. Finally, we note that the derivation of the above GLR decision rule applies to any fading distribution, and since the channel gains of the orthogonal signals are treated separately, this receiver can be applied to frequency-selective FSK systems, for example. The analysis below, however, considers the receiver's performance in the special case of frequency-flat Rayleigh fading channels.

\section{ERror Probability Bounds}

In this section, we derive upper and lower bounds on the bit error probability of the GLR test receiver for the case of a single relay, binary signaling, and flat Rayleigh fading. We also consider its asymptotic performance for large SNR.

\section{A. Conditional Error Probability}

We begin with the error probability conditioned on the relay-destination channel amplitude and later average over this variate. From (11) with $N=1$ (single relay) and $M=2$ (binary signaling), the bit error probability given $\mathrm{x}_{2}$ was sent is

$$
\begin{aligned}
P_{2} & \left(\mathcal{E} \mid \mathbf{x}_{2}, a_{1}\right) \\
& =\operatorname{Pr}\left[\frac{w_{0,2}^{2}}{\sigma^{2}}+\log w_{1,2}^{2}<\frac{w_{0,1}^{2}}{\sigma^{2}}+\log w_{1,1}^{2} \mid \mathbf{x}_{2}, a_{1}\right]
\end{aligned}
$$

where $a_{1} \triangleq a_{1,1}=a_{1,2}$ is the relay-destination channel amplitude. For equal energy signals, (12) is also the error probability given $\mathbf{x}_{1}$ was sent, and for equiprobable signals, it is the error probability conditioned on just $a_{1}$.

Using substitutions $U_{0}=w_{0,1}^{2} / \sigma^{2}, U_{1}=w_{0,2}^{2} / \sigma^{2}, V_{0}=$ $w_{1,1}^{2} / \sigma^{2}$, and $V_{1}=w_{1,2}^{2} / \sigma^{2}$ to simplify notation, (12) can be rewritten as

$$
P_{2}\left(\mathcal{E} \mid a_{1}\right)=\operatorname{Pr}\left[U_{1}-U_{0}<\log \frac{V_{0}}{V_{1}}\right] .
$$

where, due to the Rayleigh fading and Gaussian noise assumptions, the channel outputs $\mathbf{y}_{0}$ and $\mathbf{y}_{1}$ are complex Gaussian, and each variate in (13) is exponentially distributed with means

$$
\begin{aligned}
& \lambda_{0}^{-1} \triangleq \mathrm{E}\left[U_{0}\right]=1 \\
& \lambda_{1}^{-1} \triangleq \mathrm{E}\left[U_{1}\right]=\gamma_{0}+1 \\
& \nu_{0}^{-1} \triangleq \mathrm{E}\left[V_{0}\right]=\beta_{1}^{2} a_{1}^{2}+1 \\
& \nu_{1}^{-1} \triangleq \mathrm{E}\left[V_{1}\right]=\beta_{1}^{2} a_{1}^{2} \gamma_{2}+\beta_{1}^{2} a_{1}^{2}+1
\end{aligned}
$$

where $\gamma_{l}=E_{s} \mathrm{E}\left[a_{l}^{2}\right] / \sigma^{2}$ is the average SNR of channel $l$.

To assist with evaluating (13), we first present two lemmas.

Lemma 1: If $U_{0}$ and $U_{1}$ are independent and exponentially distributed with means $\lambda_{0}^{-1}$ and $\lambda_{1}^{-1}$, respectively, then the density of the difference $U_{1}-U_{0}$ is

$$
f(x)=\left\{\begin{array}{ll}
\frac{\lambda_{0} \lambda_{1}}{\lambda_{0}+\lambda_{1}} e^{-\lambda_{1} x} & ; \quad x \geq 0 \\
\frac{\lambda_{0} \lambda_{1}}{\lambda_{0}+\lambda_{1}} e^{\lambda_{0} x} & ; \quad x<0
\end{array} .\right.
$$

Lemma 1 is easily proved by convolving the densities of $U_{1}$ and $-U_{0}$.

Lemma 2: If $V_{0}$ and $V_{1}$ are independent and exponentially distributed with means $\nu_{0}^{-1}$ and $\nu_{1}^{-1}$, respectively, then the density of the natural logarithm of their ratio, $\log \left(V_{0} / V_{1}\right)$, is

$$
f(x)=\frac{\nu_{0} \nu_{1} e^{-x}}{\left(\nu_{0}+\nu_{1} e^{-x}\right)^{2}} \quad ; \quad-\infty<x<\infty .
$$

Lemma 2 can be proved by first finding the density of $Y_{i}=$ $\log V_{i}$ to be $f_{Y_{i}}(y)=\nu_{i} \exp \left(y-\nu_{i} e^{y}\right)$, then convolving the densities of $Y_{0}$ and $-Y_{1}$.

Letting $W=U_{1}-U_{0}$ and $Z=\log \left(V_{0} / V_{1}\right)$, then the conditional error probability (13) can be expressed as

$$
\begin{aligned}
P_{2}\left(\mathcal{E} \mid a_{1}\right) & =\operatorname{Pr}[W<Z] \\
& =\mathrm{E}_{Z}\{\operatorname{Pr}[W<z \mid Z=z]\}
\end{aligned}
$$


where, using Lemma 1,

$$
\begin{aligned}
\operatorname{Pr} & {[W<z \mid Z=z]=\int_{-\infty}^{z} f_{W}(w) d w } \\
& = \begin{cases}\int_{\overline{-} \infty}^{0} \frac{\lambda_{0} \lambda_{1}}{\lambda_{0}+\lambda_{1}} e^{\lambda_{0} w} d w+\int_{0}^{z} \frac{\lambda_{0} \lambda_{1}}{\lambda_{0}+\lambda_{1}} e^{-\lambda_{1} w} d w ; z \geq 0 \\
\int_{-\infty}^{z \frac{\lambda_{0} \lambda_{1}}{\lambda_{0}+\lambda_{1}} e^{\lambda_{0} w} d w}\end{cases} \\
& = \begin{cases}1-\frac{\lambda_{0}}{\lambda_{0}+\lambda_{1}} e^{-\lambda_{1} z} & ; z \geq 0 \\
\frac{\lambda_{1}}{\lambda_{0}+\lambda_{1}} e^{\lambda_{0} z} & ; \quad z<0\end{cases}
\end{aligned}
$$

Using (19) in (18) and Lemma 2,

$$
\begin{aligned}
P_{2}\left(\mathcal{E} \mid a_{1}\right)= & \int_{-\infty}^{\infty} \operatorname{Pr}[W<z \mid Z=z] f_{Z}(z) d z \\
= & \int_{-\infty}^{0} \frac{\lambda_{1}}{\lambda_{0}+\lambda_{1}} e^{\lambda_{0} z} \frac{\nu_{0} \nu_{1} e^{-z}}{\left(\nu_{0}+\nu_{1} e^{-z}\right)^{2}} d z \\
& +\int_{0}^{\infty}\left(1-\frac{\lambda_{0}}{\lambda_{0}+\lambda_{1}} e^{-\lambda_{1} z}\right) \frac{\nu_{0} \nu_{1} e^{-z}}{\left(\nu_{0}+\nu_{1} e^{-z}\right)^{2}} d z \\
= & \int_{0}^{\infty} \frac{\lambda_{1}}{\lambda_{0}+\lambda_{1}} \cdot \frac{\nu_{0} \nu_{1} e^{-\left(\lambda_{0}+1\right) z}}{\left(\nu_{1}+\nu_{0} e^{-z}\right)^{2}} d z \\
& +\int_{0}^{\infty} \frac{\nu_{0} \nu_{1} e^{-z}}{\left(\nu_{0}+\nu_{1} e^{-z}\right)^{2}} d z \\
& -\int_{0}^{\infty} \frac{\lambda_{0}}{\lambda_{0}+\lambda_{1}} \cdot \frac{\nu_{0} \nu_{1} e^{-\left(\lambda_{1}+1\right) z}}{\left(\nu_{0}+\nu_{1} e^{-z}\right)^{2}} d z
\end{aligned}
$$

Referring to the terms in (20)-(22) as $\mathcal{A}, \mathcal{B}$, and $\mathcal{C}$, respectively, then recalling that $\lambda_{0}=1$ (14) and using a change of variable for $\left(\nu_{0} / \nu_{1}\right) e^{-z}$, the first term evaluates to

$$
\mathcal{A}=\frac{\lambda_{1}}{1+\lambda_{1}} \cdot \frac{\nu_{1}}{\nu_{0}}\left[\log \left(1+\frac{\nu_{0}}{\nu_{1}}\right)-\frac{\nu_{0}}{\nu_{0}+\nu_{1}}\right] .
$$

Similarly, using a change of variable for $\left(\nu_{1} / \nu_{0}\right) e^{-z}$, the second term evaluates to $\mathcal{B}=\nu_{1} /\left(\nu_{0}+\nu_{1}\right)$.

Finally, with the same change of variable and using $[8$, (3.194.1)], the third term can be expressed as

$$
\begin{aligned}
\mathcal{C} & =\frac{1}{1+\lambda_{1}} \cdot \frac{\nu_{1} / \nu_{0}}{1+\lambda_{1}} \mathrm{~F}\left(2, \lambda_{1}+1 ; \lambda_{1}+2 ;-\nu_{1} / \nu_{0}\right) \\
& =\frac{1}{1+\lambda_{1}} \sum_{k=1}^{\infty} \frac{k}{\lambda_{1}+k}\left(-\frac{\nu_{1}}{\nu_{0}}\right)^{k}
\end{aligned}
$$

where $\mathrm{F}(\alpha, \beta ; \gamma ; z)$ is the Gauss hypergeometric function, and (23) was obtained using its series definition [8, (9.100)] and simplifying.

Combining these three terms, the conditional probability of error can be written as

$$
\begin{aligned}
& P_{2}\left(\mathcal{E} \mid a_{1}\right)=\mathcal{A}+\mathcal{B}+\mathcal{C} \\
& =\frac{\lambda_{1}}{1+\lambda_{1}} \cdot \frac{\nu_{1}}{\nu_{0}} \log \left(1+\frac{\nu_{0}}{\nu_{1}}\right) \\
& \quad+\frac{1}{1+\lambda_{1}}\left[\frac{\nu_{1}}{\nu_{0}+\nu_{1}}+\sum_{k=1}^{\infty} \frac{k}{\lambda_{1}+k}\left(-\frac{\nu_{1}}{\nu_{0}}\right)^{k}\right]
\end{aligned}
$$

Noting that $\nu_{1} / \nu_{0}<1$, the first term in the brackets in (24) can be written in the form of the power series $-\sum_{k=1}^{\infty}\left(-\nu_{1} / \nu_{0}\right)^{k}$.
Combining the two series in brackets yields

$$
\begin{aligned}
P_{2}\left(\mathcal{E} \mid a_{1}\right) & \\
= & \frac{\lambda_{1}}{1+\lambda_{1}} \cdot \frac{\nu_{1}}{\nu_{0}} \log \left(1+\frac{\nu_{0}}{\nu_{1}}\right) \\
& +\frac{1}{1+\lambda_{1}} \sum_{k=1}^{\infty}\left(\frac{k}{\lambda_{1}+k}-1\right)\left(-\frac{\nu_{1}}{\nu_{0}}\right)^{k} \\
= & \frac{\lambda_{1}}{1+\lambda_{1}} \cdot \frac{\nu_{1}}{\nu_{0}} \log \left(1+\frac{\nu_{0}}{\nu_{1}}\right) \\
& +\frac{1}{1+\lambda_{1}} \sum_{k=1}^{\infty}\left(-\frac{\lambda_{1}}{\lambda_{1}+k}\right)\left(-\frac{\nu_{1}}{\nu_{0}}\right)^{k} .
\end{aligned}
$$

An upper bound on the conditional error probability can be obtained by neglecting all but the first (positive) term in the alternating convergent series in (25). The resulting upper bound is

$$
P_{2}\left(\mathcal{E} \mid a_{1}\right)<\frac{\lambda_{1}}{1+\lambda_{1}} \cdot \frac{\nu_{1}}{\nu_{0}} \log \left(1+\frac{\nu_{0}}{\nu_{1}}\right)+\frac{\lambda_{1}}{\left(1+\lambda_{1}\right)^{2}} \cdot \frac{\nu_{1}}{\nu_{0}} .
$$

A lower bound can be obtained by replacing the divisor $\left(\lambda_{1}+k\right)$ in the series in (25) with $\left(\lambda_{1}+1\right)$, increasing the magnitude of the negative partial series that begins with the second term. The resulting lower bound is

$$
P_{2}\left(\mathcal{E} \mid a_{1}\right)>\frac{\lambda_{1}}{1+\lambda_{1}} \cdot \frac{\nu_{1}}{\nu_{0}} \log \left(1+\frac{\nu_{0}}{\nu_{1}}\right)+\frac{\lambda_{1}}{\left(1+\lambda_{1}\right)^{2}} \cdot \frac{\nu_{1}}{\nu_{0}+\nu_{1}} .
$$

\section{B. Error Probability Upper Bound}

Using the bounds on the conditional error probability derived above, we wish to develop bounds on the unconditional error probability, removing the conditioning on the relaydestination channel amplitude, $a_{1}$. Of the quantities in (26) and (27), only $\nu_{0}$ and $\nu_{1}$ are a function of $a_{1}$.

Starting with the upper bound (26) and taking its expectation with respect to $a_{1}$, the unconditional error probability is upper bounded by

$P_{2}(\mathcal{E})<\frac{\lambda_{1}}{1+\lambda_{1}} \mathrm{E}\left[\frac{\nu_{1}}{\nu_{0}} \log \left(1+\frac{\nu_{0}}{\nu_{1}}\right)\right]+\frac{\lambda_{1}}{\left(1+\lambda_{1}\right)^{2}} \mathrm{E}\left[\frac{\nu_{1}}{\nu_{0}}\right]$.

Since $x \log (1+1 / x)$ is a concave function of $x$, then using Jensen's inequality the first expectation in (28) can be upper bounded as

$$
\mathrm{E}\left[\frac{\nu_{1}}{\nu_{0}} \log \left(1+\frac{\nu_{0}}{\nu_{1}}\right)\right] \leq \mathrm{E}\left[\frac{\nu_{1}}{\nu_{0}}\right] \log \left(1+\frac{1}{\mathrm{E}\left[\nu_{1} / \nu_{0}\right]}\right) .
$$

Thus, only the expectation $\mathrm{E}\left[\nu_{1} / \nu_{0}\right]$ is needed for the resulting error probability upper bound.

From (16) and (17), we have

$$
\frac{\nu_{1}}{\nu_{0}}=\frac{\beta_{1}^{2} a_{1}^{2}+1}{\beta_{1}^{2}\left(\gamma_{2}+1\right) a_{1}^{2}+1} .
$$

Under the assumption of Rayleigh fading, $a_{1}^{2}$ is exponentially distributed. Let $\eta_{1}^{-1}$ be the mean of $a_{1}^{2}$. Then averaging (30) 
over $a_{1}^{2}$ yields

$$
\begin{aligned}
\mathrm{E}\left[\frac{\nu_{1}}{\nu_{0}}\right]= & \int_{0}^{\infty} \frac{\beta_{1}^{2} x+1}{\beta_{1}^{2}\left(\gamma_{2}+1\right) x+1} \eta_{1} e^{-\eta_{1} x} d x \\
= & \frac{\eta_{1} \gamma_{2}}{\beta_{1}^{2}\left(\gamma_{2}+1\right)^{2}} \exp \left(\frac{\eta_{1}}{\beta_{1}^{2}\left(\gamma_{2}+1\right)}\right) \\
& \times \mathrm{E}_{1}\left(\frac{\eta_{1}}{\beta_{1}^{2}\left(\gamma_{2}+1\right)}\right)+\frac{1}{\gamma_{2}+1}
\end{aligned}
$$

where $[8,(3.352 .4)$ and $(3.353 .5)]$ were used to evaluate the integral, and where $\mathrm{E}_{1}(\cdot)$ is a form of the exponential integral.

Using the following bounds on $e^{x} \mathrm{E}_{1}(x)$ [9, (5.1.20)],

$$
\frac{1}{2} \log \left(1+\frac{2}{x}\right)<e^{x} \mathrm{E}_{1}(x)<\log \left(1+\frac{1}{x}\right)
$$

(31) is bounded by

$\mathrm{E}\left[\frac{\nu_{1}}{\nu_{0}}\right]<\frac{\eta_{1} \gamma_{2}}{\beta_{1}^{2}\left(\gamma_{2}+1\right)^{2}} \log \left(1+\frac{\beta_{1}^{2}\left(\gamma_{2}+1\right)}{\eta_{1}}\right)+\frac{1}{\gamma_{2}+1}$

$\mathrm{E}\left[\frac{\nu_{1}}{\nu_{0}}\right]>\frac{\eta_{1} \gamma_{2}}{2 \beta_{1}^{2}\left(\gamma_{2}+1\right)^{2}} \log \left(1+\frac{2 \beta_{1}^{2}\left(\gamma_{2}+1\right)}{\eta_{1}}\right)+\frac{1}{\gamma_{2}+1}$.

Denoting these upper and lower bounds on $\mathrm{E}\left[\nu_{1} / \nu_{0}\right]$ by $\mu_{0, \mathrm{ub}}$ and $\mu_{0, \mathrm{lb}}$, respectively, and using them with (28) and (29), the error probability can be further upper bounded by

$$
P_{2}(\mathcal{E})<\frac{\lambda_{1}}{1+\lambda_{1}} \mu_{0, \mathrm{ub}} \log \left(1+\frac{1}{\mu_{0, \mathrm{lb}}}\right)+\frac{\lambda_{1}}{\left(1+\lambda_{1}\right)^{2}} \mu_{0, \mathrm{ub}}
$$

\section{Error Probability Lower Bound}

To obtain a lower bound on the unconditional error probability, we take the expectation of (27) with respect to $a_{1}^{2}$ :

$$
\begin{aligned}
P_{2}(\mathcal{E})> & \frac{\lambda_{1}}{1+\lambda_{1}} \mathrm{E}\left[\frac{\nu_{1}}{\nu_{0}} \log \left(1+\frac{\nu_{0}}{\nu_{1}}\right)\right] \\
& +\frac{\lambda_{1}}{\left(1+\lambda_{1}\right)^{2}} \mathrm{E}\left[\frac{\nu_{1}}{\nu_{0}+\nu_{1}}\right] .
\end{aligned}
$$

Since $(1 / x) \log (1+x)$ is a convex function of $x$, then using Jensen's inequality again, the first expectation in (33) can be lower bounded as

$$
\mathrm{E}\left[\frac{\nu_{1}}{\nu_{0}} \log \left(1+\frac{\nu_{0}}{\nu_{1}}\right)\right] \geq \frac{1}{\mathrm{E}\left[\nu_{0} / \nu_{1}\right]} \log \left(1+\mathrm{E}\left[\frac{\nu_{0}}{\nu_{1}}\right]\right) .
$$

Repeating the procedure given in Section IV-B, bounds on $\mathrm{E}\left[\nu_{0} / \nu_{1}\right]$ are found to be

$$
\begin{aligned}
& \mathrm{E}\left[\frac{\nu_{0}}{\nu_{1}}\right]<\gamma_{2}\left[1-\frac{\eta_{1}}{2 \beta_{1}^{2}} \log \left(1+\frac{2 \beta_{1}^{2}}{\eta_{1}}\right)\right]+1 \\
& \mathrm{E}\left[\frac{\nu_{0}}{\nu_{1}}\right]>\gamma_{2}\left[1-\frac{\eta_{1}}{\beta_{1}^{2}} \log \left(1+\frac{\beta_{1}^{2}}{\eta_{1}}\right)\right]+1
\end{aligned}
$$

which we denote as $\mu_{1, \mathrm{ub}}$ and $\mu_{1, \mathrm{lb}}$, respectively.

Likewise, a lower bound of $\mathrm{E}\left[\nu_{1} /\left(\nu_{0}+\nu_{1}\right)\right]$ can be found as

$$
\begin{aligned}
& \mathrm{E}\left[\frac{\nu_{1}}{\nu_{0}+\nu_{1}}\right] \\
& \quad>\frac{\eta_{1} \gamma_{2}}{2 \beta_{1}^{2}\left(\gamma_{2}+2\right)^{2}} \log \left(1+\frac{\beta_{1}^{2}\left(\gamma_{2}+2\right)}{\eta_{1}}\right)+\frac{1}{\gamma_{2}+2}
\end{aligned}
$$

denoted by $\mu_{2, \mathrm{lb}}$.

Finally, using $\mu_{1, \mathrm{ub}}, \mu_{1, \mathrm{lb}}$, and $\mu_{2, \mathrm{lb}}$ with (33) and (34), the error probability is further lower bounded by

$$
P_{2}(\mathcal{E})>\frac{\lambda_{1}}{1+\lambda_{1}} \cdot \frac{1}{\mu_{1, \mathrm{ub}}} \log \left(1+\mu_{1, \mathrm{lb}}\right)+\frac{\lambda_{1}}{\left(1+\lambda_{1}\right)^{2}} \mu_{2, \mathrm{lb}} .
$$

\section{Asymptotic Performance}

Here, we estimate the error probability upper bound for large SNR to analyze the asymptotic performance of the single-relay binary receiver in Rayleigh fading channels. Let $\eta_{l}^{-1}=\mathrm{E}\left[a_{l}^{2}\right]$ be the average channel gain of link $l$ and let $\gamma=E_{s} / \sigma^{2}$, so that $\gamma_{l}=\gamma / \eta_{l}$. Recalling that $\lambda_{1}^{-1}=\gamma_{0}+1$ (15), the error probability upper bound (32) can be approximated for large $\gamma$ as

$$
P_{2, \mathrm{ub}}(\mathcal{E}) \approx \frac{\eta_{0}}{\gamma} \mu_{0, \mathrm{ub}}\left[\log \left(1+\frac{1}{\mu_{0, \mathrm{lb}}}\right)+1\right] .
$$

Using $(1 / x) \log (1+x)>1 /(1+x), \mu_{0, \mathrm{lb}}$ can be further lower bounded as

$$
\mu_{0, \mathrm{lb}}>\frac{1}{\gamma_{2}+1}\left[\frac{\gamma_{2}}{1+2 \beta_{1}^{2}\left(1+\gamma_{2}\right) / \eta_{1}}+1\right] \triangleq \mu_{0, \mathrm{lb}}^{\prime} .
$$

For large $\gamma$, this lower bound can be approximated as

$$
\begin{aligned}
\mu_{0, \mathrm{lb}}^{\prime} & \approx \frac{1}{\gamma_{2}}\left(\frac{\eta_{1}}{2 \beta_{1}^{2}}+1\right) \\
& \approx \frac{1}{\gamma}\left(\frac{\eta_{1}}{2}+\eta_{2}\right)
\end{aligned}
$$

where in the second line we assume the fixed relay gain (3), for which $\beta_{1}^{2}=1 /\left(\eta_{2}^{-1}+2 / \gamma\right) \approx \eta_{2}$ for large $\gamma$.

The upper bound $\mu_{0, \mathrm{ub}}$ can be approximated as

$$
\begin{aligned}
\mu_{0, \mathrm{ub}} & \approx \frac{1}{\gamma_{2}}\left(\frac{\eta_{1}}{\beta_{1}^{2}} \log \frac{\beta_{1}^{2} \gamma_{2}}{\eta_{1}}+1\right) \\
& \approx \frac{1}{\gamma}\left(\eta_{1} \log \frac{\gamma}{\eta_{1}}+\eta_{2}\right) .
\end{aligned}
$$

Using (37) and (38) in (36), the upper bound of the error probability can be approximated for large SNR as

$$
\begin{aligned}
& P_{2, \mathrm{ub}}(\mathcal{E}) \\
& \quad \approx \frac{\eta_{0}}{\gamma^{2}}\left(\eta_{1} \log \frac{\gamma}{\eta_{1}}+\eta_{2}\right)\left[\log \left(1+\frac{\gamma}{\eta_{1} / 2+\eta_{2}}\right)+1\right] \\
& \approx \frac{\eta_{0}}{\gamma^{2}}\left(\eta_{1} \log \frac{\gamma}{\eta_{1}}+\eta_{2}\right) \log \left(\frac{\gamma}{\eta_{1} / 2+\eta_{2}}\right) \\
& \quad \sim \mathcal{O}\left(\frac{\log ^{2} \gamma}{\gamma^{2}}\right) .
\end{aligned}
$$

Thus, we observe that the error probability of the noncoherent binary GLR test receiver decreases with SNR as $\log ^{2}$ (SNR) $/ \mathrm{SNR}^{2}$ at high SNR. Since $\log x$ increases much more slowly than $x$, this receiver achieves near second-order diversity with a single relay in Rayleigh fading. Interestingly, by comparison, the bit error probability of the differential modulation receiver in [6] decays as $\log (\mathrm{SNR}) / \mathrm{SNR}^{2}$, though this receiver requires knowledge of the average source-relay and relay-destination channel gains. 


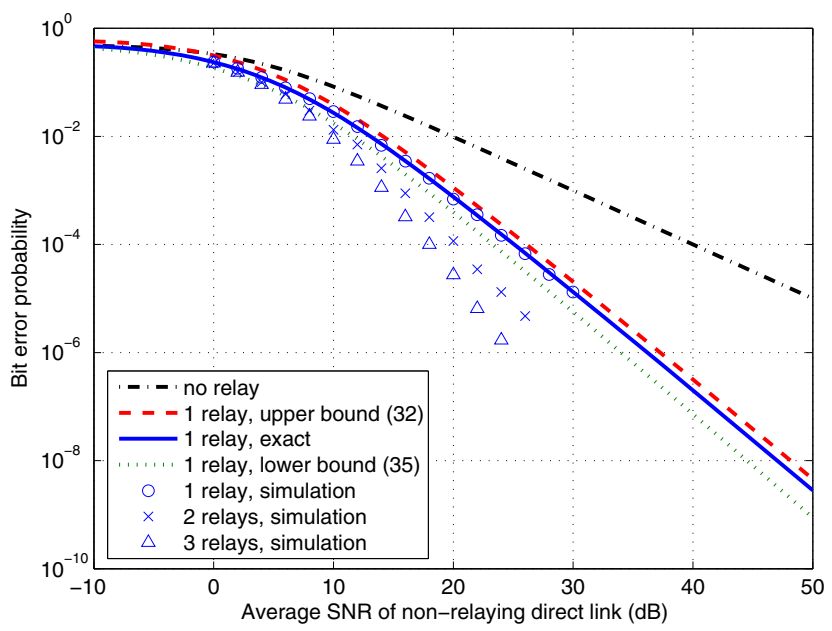

Fig. 2. BER vs. SNR, $M=2$, all relays at midpoint

\section{NumericAl Results}

Examples are given of the receiver's performance in flat Rayleigh fading using the probability of error expressions developed in the previous section as well as results obtained by Monte Carlo simulation. The average signal strength is assumed to decrease with the fourth-power of distance, so that $\eta_{l}=d_{l}^{4}$ where $d_{l}$ is the distance of link $l(l=0, \ldots, 2 N)$. For the results below, $d_{0}$ is normalized to unity, and $\left\{d_{l}\right\}, l>0$, are varied. Results are given with respect to the average SNR in a non-relaying system using the same total energy, equally divided among the source and relays in the relaying system.

Fig. 2 illustrates the bit error rate (BER) versus the SNR per bit with binary signaling for the non-relaying sourcedestination link as well as for several relaying scenarios in which the relays are placed at the midpoint between the source and destination. The exact bit error probability for the case of one relay is obtained by numerically evaluating the expectation of (24) with respect to the relay-destination channel gain. The upper and lower bounds are plotted using the closedform expressions (32) and (35), respectively. We observe the simulation results to be in agreement with the predicted results. Additional simulation results for two and three relays demonstrate increasing diversity gain with additional relays. Comparing with similar results in [5], the GLR test receiver performs only about $1.5 \mathrm{~dB}$ from the higher complexity optimum binary FSK receiver at a BER of $4 \times 10^{-4}$.

The preceding results assume use of fixed relay gain (3) representing a long-term power constraint at the relay. Simulation results with variable gain (2), not shown here for brevity, indicate a $2 \mathrm{~dB}$ to $3 \mathrm{~dB}$ improvement at $10^{-5}$ BER with 1-3 relays. Thus, the expressions derived above appear to be useful as upper bounds on the performance with an instantaneous power constraint at the relay rather than a long-term constraint.

Next, the position of a single relay is varied along the line between the source and destination. Fig. 3 plots the BER as a function of the source-relay distance. The bounds are observed to become increasingly tight as the relay moves closer to the destination. Furthermore, the optimum position of the relay

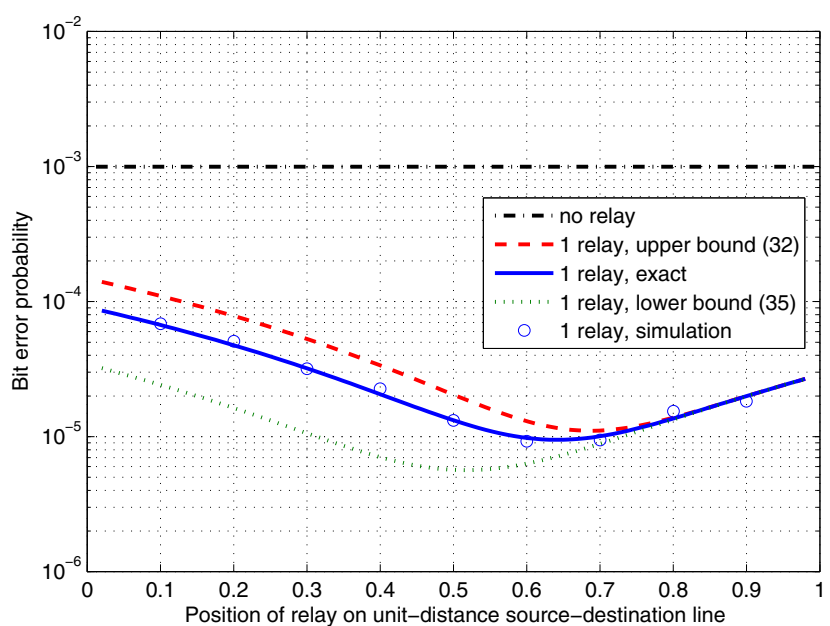

Fig. 3. BER vs. relay position, one relay, $M=2$, SNR $=30 \mathrm{~dB}$

is at 0.64 of the source-destination distance for a path-loss exponent of four. With coherent receivers, by contrast, the optimum relay position is at the midpoint [3].

\section{CONCLUSION}

We have found a low-complexity, non-coherent amplifyand-forward receiver that requires no statistical knowledge of the channel gains in the system. The decision metric was obtained using the generalized likelihood ratio test. Bounds on the bit error probability were derived in closed form for the case of binary signals, a single relay, and Rayleigh fading channels, and the error probability of the receiver was shown to decrease as $\log ^{2}$ (SNR) $/ \mathrm{SNR}^{2}$, approaching full, second-order diversity with increasing SNR. Simulation results showed increased diversity gain with additional relays. While the analysis focused on Rayleigh fading, the derived decision metric is, in fact, independent of the fading distribution.

\section{REFERENCES}

[1] J. N. Laneman and G. W. Wornell, "Energy-efficient antenna sharing and relaying for wireless networks," in Proc. IEEE Wireless Communications and Networking Conference (WCNC), Sep. 2000.

[2] A. Ribeiro, X. Cai, and G. B. Giannakis, "Symbol error probabilities for general cooperative links," IEEE Trans. Wireless Commun., vol. 4, no. 3, pp. 1264-1273, May 2005.

[3] M. R. Souryal and B. R. Vojcic, "Performance of amplify-and-forward and decode-and-forward relaying in Rayleigh fading with turbo codes," in Proc. IEEE Int. Conf. Acoustics, Speech, and Signal Processing (ICASSP), vol. 4, May 2006, pp. 681-684.

[4] D. Chen and J. N. Laneman, "Cooperative diversity for wireless fading channels without channel state information," in Proc. Asilomar Conf. Signals, Syst. and Comp., vol. 2, Nov. 2004, pp. 1307-1312.

[5] R. Annavajjala, P. C. Cosman, and L. B. Miltstein, "On the performance of optimum noncoherent amplify-and-forward reception for cooperative diversity," in Proc. MILCOM, vol. 5, Oct. 2005.

[6] Q. Zhao and H. Li, "Performance of differential modulation with wireless relays in Rayleigh fading channels," IEEE Commun. Lett., vol. 9, no. 4, pp. 343-345, Apr. 2005.

[7] H. L. Van Trees, Detection, Estimation, and Modulation Theory: Part I. New York: John Wiley and Sons, Inc., 1968.

[8] I. S. Gradshteyn and I. M. Ryzhik, Table of Integrals, Series and Products. 6th ed., New York: Academic Press, 2000.

[9] M. Abramowitz and I. A. Stegun, Eds., Handbook of Mathematical Functions. 9th ed., Washington, DC: U.S. Department of Commerce, National Bureau of Standards, 1970. 